\title{
Sodium Phenylbutyrate
}

National Cancer Institute

\section{Source}

National Cancer Institute. Sodium Phenylbutyrate. NCI Thesaurus. Code C1440.

The sodium salt of phenylbutyrate, a derivative of the short-chain fatty acid butyrate,

with potential antineoplastic activity. Phenylbutyrate reversibly inhibits class I and II

histone deacetylases (HDACs), which may result in a global increase in gene expression,

decreased cellular proliferation, increased cell differentiation, and the induction of apoptosis in susceptible tumor cell populations. 